\title{
NuEVAS PUBLICACIONES Y REIMPRESIONES (2016)
}

Backhouse, Stephen. Kierkegaard: A Single Life. Michigan: Zondervan, 2016.

Barnett, Christopher B. Kierkegaard, Pietism and Holiness. London: Routledge, 2016.

Benavides, Cristian Eduardo. Libertad, realidad y posibilidad en Begrebet Angest Un estudio acerca de las categorías existenciales de Søren Kierkegaard en relación con la angustia. Saarbrücken: Editorial Académica Española, 2016.

Bentivegna, Maria Elena. Kierkegaard. Bologna: Diogene multimedia, 2016.

Blättel, Richard. Das Geheimnis der Wiederbolung Sören Kierkegaard passiert jüdisches Denken. Deutschland: Transcript, 2016.

Brøchner, Hans, Carl Henrik Koch, and Kongelige Danske Videnskabernes Selskab. Hans Brøchners bidrag til Kierkegaard-receptionen i Danmark. Københavns: Det Kongelige Danske Videnskabernes Selskab, 2016.

Buben, Adam. Meaning and Mortality in Kierkegaard and Heidegger: Origins of the Existential Philosophy of Death. Illinois: Northwestern University Press, 2016.

Calfapietra, Lucia, Line Faden-Babin, Jakob Rachmanski, Thomas Laugstien, and Diaphanes Verlag. Kierkegaard und die Meerjungfrau. Zürich: Diaphanes, 2016.

Chaning-Pearce, Melville. Terrible Crystal: Studies in Kierkegaard and Modern Christianity. London: Routledge, 2016.

Collins, James Daniel. Mind of Kierkegaard. New Jersey: Princeton University Pres, 2016.

Connell, George. Kierkegaard and the Paradox of Religious Diversity. Michigan: Eerdmans Publishing Company, 2016.

Dalager, Stig. L'uomo dell'istante: un romanzo su Søren Kierkegaard. Milano: Iperborea, 2016.

Deuser, Hermann, and Markus Kleinert. Søren Kierkegaard: Entweder - Oder. Berlin: De Gruyter, 2016.

Duncan, Elmer H. Søren Kierkegaard. Massachusetts: Hendrickson Publishers, 2016. 
Dunning, Stephen Northrup. Kierkegaard's Dialectic of Inwardness. New Jersey: Princeton University Pres, 2016.

Eller, Vernard. Kierkegaard and Radical Discipleship. New Jersey: Princeton University Press, 2016.

Elrod, John W. Being and Existence in Kierkegaard's Pseudonymous Works. New Jersey: Princeton University Pres, 2016. 2016.

Faden-Babin, Line, and Jakob Rachmanski. Kierkegaard og havfruen. Farum: Arvids, 2016.

Frazier, B. Rorty and Kierkegaard on Irony and Moral Commitment: Philosophical and Theological Connections. London: Palgrave Macmillan, 2016.

Furnal, Joshua. Catholic Theology after Kierkegaard. Oxford: Oxford University Press, 2016.

Gimenes de Paula, Marcio. Kierkegaard em diálogo com a tradição filosófica. São Paulo: Intermeios, 2016.

Golomb, Jacob. In Search of Authenticity: Existentialism from Kierkegaard to Camus. London: Taylor \& Francis, 2016.

González, Darío, Laura Llevadot Pascual, and Begonya Sáez Tajafuerce. Kierkegaard y las artes. Barcelona: Editorial UOC, 2016.

Grünling, Markus. Sören Kierkegaard - Die philosophische Supernova aus dem Norden Einführungsvortrag in Leben und Denken. Wildflecken: Azur Verlag, 2016.

Jothen, Peder. Kierkegaard, Aesthetics, and Selfhood the Art of Subjectivity. London: Routledge, 2016.

Kierkegaard, Søren. Kierkegaard's Journals and Notebooks, Volume 9, Journals nb26-nb30. New Jersey: Princeton University Pres, 2016.

Kierkegaard, Søren. Mi Punto de Vista, trad. José Miguel Velloso. Ciudad de México: Fontamara, 2016.

Kierkegaard, Søren, Darío González, and Óscar Parcero Oubiña. Migajas filosóficas; El concepto de angustia; Prólogos. Madrid: Trotta, 2016.

Krishek, Sharon. Kierkegaard on Faith and Love. Cambridge: Cambridge University Press, 2016. 
Kuder, Paul, and Nomos Verlagsgesellschaft. Heideggers Kierkegaard. BadenBaden: Nomos, 2016.

Lippitt, John. Humor and Irony in Kierkegaard's Thought. London: Palgrave Macmillan, 2016.

The Routledge Guidebook to Kierkegaard's Fear and Trembling. London: Routledge, 2016.

Martens, Paul Henry, and C. Stephen Evans, eds. Kierkegaard and Cbristian Faith, 2016.

Peter Šajda \& Jon Stewart (Editors), ed. Kierkegaard Bibliography: Afrikaans to Dutch. New York: Routledge, 2016.

-Kierkegaard Bibliography: English. New York: Routledge, 2016.

-Kierkegaard Bibliography: Estonian to Hebrew. New York: Routledge, 2016.

_Kierkegaard Bibliography: Figures A to H. New York: Routledge, 2016.

_Kierkegaard Bibliography Figures I to Z. New York: Routledge, 2016.

Kierkegaard Bibliography: Latvian to Ukrainian. New York: Routledge, 2016. 2016

Kierkegaard Bibliography: Hungarian to Korean. New York: Routledge,

Rauh, Raphael Benjamin. Modulationen der Einsamkeit: Theorien der Ausnabme als Moralkritik bei Sören Kierkegaard und Friedrich Nietzsche. Alber Thesen, 2016.

Rocca, Ettore. Kierkegaard. København: Gyldendal, 2016.

Rush, Fred. Irony and Idealism: Rereading Schlegel, Hegel, and Kierkegaard. Oxford: Oxford University Press, 2016.

Shmueli, Adi. Kierkegaard and Consciousness. New Jersey: Princeton University Pres, 2016.

Söderquist, Anna Strelis. Kierkegaard on Dialogical Education: Vulnerable Freedom. Mariland: Rowman \& Littlefield Publishers, 2016.

Spanos, William V. On the Ethical Imperatives of the Interregnum Essays in Loving Strife from Soren Kierkegaard to Cornel West. London: Palgrave Macmillan, 2016.

Stewart, Jon. Kierkegaard Secondary Literature: Greek, Hebrew, Italian, Japanese, Norwegian and Polish. New York: Routledge, 2016. 
Stewart, Jon. Kierkegaard and the Bible - the Old Testament. Vol. 1, Tome I. New York: Routledge, 2016.

. Kierkegaard Secondary Literature. Catalan, Chinese, Czech, Danish and Dutch Tome I Tome I. New York: Routledge, 2016.

. Kierkegaard Secondary Literature. English, A - K Tome II Tome II. New York: Routledge, 2016.

Kierkegaard Secondary Literature. English L-Z Tome III Tome III. New York: Routledge, 2016.

Kierkegaard Secondary Literature: Finnish, French, Galician and German. New York: Routledge, 2016.

Kierkegaard Secondary Literature. Finnish, French, Galician and German Tome IV Tome IV. New York: Routledge, 2016.

- Kierkegaard Secondary Literature. Greek, Hebrew, Hungarian, Italian, Japanese, Norwegian and Polish Tome V Tome V. New York: Routledge, 2016.

- Kierkegaard Secondary Literature. Portuguese, Romanian, Russian, Slovak, Spanish and Swedish Tome VI Tome VI. New York: Routledge, 2016.

—. Kierkegaard Secondary Literature. Tome II, Tome II,. New York: Routledge, 2016.

. Kierkegaard Secondary Literature: Volume 18, Tome Ii. New York: Routledge, 2016. 2016.

. Kierkegaard's Influence on Theology. Vol. 10, Tome I. S.1.: ROUTLEDGE, . Kierkegaard's Influence on Theology. Vol. 10, Tome II. New York: Routledge, 2016.

- Kierkegaard Secondary Literature: Greek, Hebrew, Italian, Japanese, Norwegian and Polish. New York: Routledge, 2016.

- Kierkegaard Research: Sources, Reception and Resources Vol. 18 Tome I. New York: Routledge, 2016.

- Kierkegaard Research: Sources, Reception and Resources Volume 18 Tome II Volume 18 Tome 1. New York: Routledge, 2016.

- Kierkegaard Research: Sources, Reception and Resources Volume 18 Tome III Volume 18 Tome 1. New York: Routledge, 2016. 
Stokes, P. Kierkegaard's Mirrors. Interest, Self, and Moral Vision. London: Palgrave Macmillan, 2016.

Theunissen, Michael. Kierkegaard's Concept of Despair. New Jersey: Princeton University Pres, 2016.

Tietjen, Mark A. Kierkegaard: A Christian Missionary to Christians. Illinois: IVP Academic, 2016. 